\title{
Target Tracking with Random Time-delay Measurements
}

\author{
Jue Huang ${ }^{1, a}$, Bing Yan ${ }^{1, b}$, Dong Li ${ }^{2, c}$ \\ ${ }^{1}$ Department of Weaponry Engineering, Naval University of Engineering, Wuhan, Hubei 430033, \\ People's Republic of China \\ ${ }^{2}$ College of science, Naval University of Engineering, Wuhan, Hubei 430033, People's Republic of \\ China \\ anudt_huangjue@163.com, ${ }^{\mathrm{b}}$ yanbing_wuhan@yeah.net, ${ }^{\mathrm{c}}$ lidong@163.com
}

Keywords: target tracking; delayed measurements; Kalman Filter; current state; Monte Carlo simulations.

\begin{abstract}
The problem of target tracking with the delayed measurements is investigated in this paper. In many practical applications, the measurements may be randomly delayed. In these cases, the standard Kalman Filter cannot be used directly to estimate current states. A novel algorithm based on the delayed measurement is proposed to estimate the current states. In the algorithm, the equivalent delayed measurement is presented as a function of the current state. Then the Kalman Filter which the process noise and the measurement noise are correlative has been deduced. Monte Carlo simulations have been designed and the results verify the effectiveness of the algorithm.
\end{abstract}

\section{Introduction}

In recent years, state estimation has attached considerable attention due to its wide applications in target tracking, communication, control, and so on. Many filter methods are commonly used such as the Kalman filter, the extended Kalman filter and the particle filter. However, all of the algorithms above are based on the assumption that the measurements are transmitted to the filter without any delay. But in practice, this assumption may be wrong. In fact, the measurement delays exist more or less in all of the systems. When the measurement is delayed, the current state cannot be corrected directly by the current measurement. That's because the delayed measurement usually carries the information of a past state. For the delayed measurements problem, a set of solutions have been proposed. Bar Shalom ${ }^{[1]}$ proposed an optimal solution and sub-solutions for one-step delayed measurements. In [2], an algorithm for the multiple-step delayed measurements was proposed. Challa $^{[3]}$ proposed a Bayesian solution for the out-of-sequence measurements problem. In [4], Lu has proposed a Kalman filtering for time-delayed linear system. And Zhang applied the out-of-sequence to the distributed fusion system ${ }^{[5]}$. Another solution is to estimate the corresponding past state using the delayed measurement, and then the current state is predicted by the past state. This algorithm can be described as augment state form ${ }^{[6]}$. The augment state is to collect the states in several steps as a new state, which will contain both the current state and the past state corresponding to the delayed measurement. Merwe ${ }^{[7]}$ applied the augment state method to the sigma point Kalman filter. However, the augmented state Kalman filter (ASKF) also has its limitation. When the dimension of the state space is very large and the number of the delayed steps is large, the dimension of the augment state will be too huge. That is a cost of storage space and the storage space may not be able to satisfy the costs sometimes.

In this paper, a kalman filter algorithm based on the equivalent measurement equation is proposed. First, the relation between the current state and the past state corresponding to the delayed measurement has been deduced through iterating the system equation. Then the equivalent measurement equation based on the current state will be built. Second, the Kalman filter which the process noise and the measurement noise are dependent is deduced. The new Kalman gain has obtained.

This paper is organized as follows. In Section 2, the mathematical model of the delayed measurement is described. In Section 3, the estimation algorithm for the delayed measurement is 
proposed and the Kalman filter with dependent noise is deduced. Then, in Section 4, the simulation example is presented. The simulation results have shown the effectiveness of the proposed algorithm. Then a conclusion is given in Section 5.

\section{Problem Statement}

In this Section, the delayed measurement problem is described. Consider the following discrete time-delay linear system:

$$
\begin{aligned}
& x(k)=A x(k-1)+w(k) \\
& y(k)=H x(k-\Delta t)+v(k-\Delta t)
\end{aligned}
$$

where $x(k) \in R^{n}$ is the state vector, $y(k) \in R^{m}$ is the measurement vector of the sensor, $A$ is the constant system matrix, $H$ is the observation matrix of the sensor, $w(k)$ is the process noise, $v(k)$ is the measurement noise, $\Delta t$ is the measurement delay. Because the delay cannot be specified exactly, it can be assumed to be a random value which can be described with a PDF.

Assume that all the noises are independent with each other and the initial state is independent to the noises.

\section{Kalman Filter for Delayed Measurement System}

This Section first reviews the basic Kalman Filter with no delayed measurements. Then the Kalman Filter for the delayed measurements is deduced.

Consider the linear system described by (1) (2), As the delay is described as a PDF, the delay is a continuous random variable. But the filter step is discrete. So the delay must be approximated. If the amount of time delay is between the filter time step $t_{k}$ and $t_{k+1}$, then the delayed time step $\Delta k$ will be considered to be $k+1$.

Consider equation (1), it can be obtained that:

$x(k)=A x(k-1)+w(k)$

$x(k-1)=A x(k-2)+w(k-2)$

$\Lambda$

$x(k-\Delta k+1)=A x(k-\Delta k)+w(k-\Delta k)$

The relation between the current state and the past state is:

$$
x(k)=A^{\Delta k} x(k-\Delta k)+A^{\Delta k-1} w(k-\Delta k)+\mathrm{L}+A^{\Delta k-m-1} w(k-\Delta k+m)+\mathrm{L}+w(k-1)
$$

Define:

$\eta(k)=A^{\Delta k-1} w(k-\Delta k)+\mathrm{L}+A^{\Delta k-m-1} w(k-\Delta k+m)+\mathrm{L}+w(k-1)$

Thus, (6) can be written as:

$$
x(k)=A^{\Delta k} x(k-\Delta k)+\eta(k)
$$

So:

$$
x(k-\Delta k)=A^{-\Delta k} x(k)-A^{-\Delta k} \eta(k)
$$

Then the delayed measurement equation can be written as:

$$
y(k)=H A^{-\Delta k} x(k)-H A^{-\Delta k} \eta(k)+v(k-\Delta k)
$$

Define:

$$
\varepsilon(k)=-H A^{-\Delta k} \eta(k)+v(k-\Delta k)
$$

Then:

$$
y(k)=H A^{-\Delta k} x(k)+\varepsilon(k)
$$

Then the delayed measurement is written as a function of the current state. Thus the Kalman Filter can be used. But equivalent measurement noise $\varepsilon(k)$ contains a part $w(k-1)$, and the process noise also contains the part $w(k-1)$, the standard Kalman Filter must be updated to adjust the situation which the noises are dependent. 
For the equivalent system (1) (12), the state predict is:

$$
\begin{aligned}
& \hat{x}(k \mid k-1)=A \hat{x}(k-1 \mid k-1) \\
& P(k \mid k-1)=A P(K-1 \mid k-1) A^{T}+Q
\end{aligned}
$$

The measurement update:

$$
\begin{aligned}
& \hat{y}(k \mid k-1)=H A^{-\Delta k} \hat{x}(k \mid k-1) \\
& \hat{x}(k \mid k)=\hat{x}(k \mid k-1)+\bar{K}(k)[y(k)-\hat{y}(k \mid k-1)]
\end{aligned}
$$

From the basic Kalman theory, it is known that the Kalman gain meets:

$$
\bar{K}(k)=R_{x_{k} y_{R}} R_{y_{R} y_{k}}^{-1}
$$

Where:

$$
\begin{aligned}
R_{x_{k} \tilde{y}_{k}} & =\operatorname{cov}(x(k), \tilde{y}(k \mid k-1))=E\left\{\tilde{x}(k \mid k-1)\left[H A^{-\Delta k} \tilde{x}(k \mid k-1)+\varepsilon(k)\right]^{T}\right\} \\
& =E\left\{\tilde{x}(k \mid k-1)\left[H A^{-\Delta k} \tilde{x}(k \mid k-1)\right]^{T}\right\}+E\left[\tilde{x}(k \mid k-1) \varepsilon^{T}(k)\right] \\
& =P(k \mid k-1)\left(H A^{-\Delta k}\right)^{T}+E\left[\tilde{x}(k \mid k-1) \varepsilon^{T}(k)\right]
\end{aligned}
$$

Known that:

$\tilde{x}(k \mid k-1)=A \tilde{x}(k-1 \mid k-1)+w(k-1)$

it can be obtained that:

$$
E\left[\tilde{x}(k \mid k-1) \varepsilon^{T}(k)\right]=-Q\left(H A^{-\Delta k}\right)^{T}
$$

Then:

$$
R_{x_{k} y_{k}}=P(k \mid k-1)\left(H A^{-\Delta k}\right)^{T}-Q\left(H A^{-\Delta k}\right)^{T}
$$

For (17):

$$
\begin{aligned}
R_{\tilde{y}_{k} \tilde{y}_{k}} & =\operatorname{cov}(\tilde{y}(k \mid k-1)) \\
& =E\left[\left(H A^{-\Delta k} \tilde{x}(k \mid k-1)+\varepsilon(k)\right)\left(H A^{-\Delta k} \tilde{x}(k \mid k-1)+\varepsilon(k)\right)^{T}\right] \\
& =E\left[\left(H A^{-\Delta k} \tilde{x}(k \mid k-1)\right)\left(H A^{-\Delta k} \tilde{x}(k \mid k-1)\right)^{T}\right] \\
& +E\left[\left(H A^{-\Delta k} \tilde{x}(k \mid k-1)\right) \varepsilon^{T}(k)\right] \\
& +E\left[\varepsilon(k)\left(H A^{-\Delta k} \tilde{x}(k \mid k-1)\right)^{T}\right]+E\left[\varepsilon(k) \varepsilon^{T}(k)\right] \\
& =H A^{-\Delta k} P(k \mid k-1)\left(H A^{-\Delta k}\right)^{T}-H A^{-\Delta k} Q^{T}-Q\left(H A^{-\Delta k}\right)^{T} \\
& +E\left[\varepsilon(k) \varepsilon^{T}(k)\right]
\end{aligned}
$$

Where:

$$
E\left[\varepsilon(k) \varepsilon^{T}(k)\right]=E\left[\left(-H A^{-\Delta k} \eta(k)+v(k-\Delta k)\right)\left(-H A^{-\Delta k} \eta(k)+v(k-\Delta k)\right)^{T}\right]
$$

It can be obtained from the dependence of the noises that:

$E\left[\varepsilon(k) \varepsilon^{T}(k)\right]=H A^{-1} Q\left(H A^{-1}\right)^{T}+H A^{-2} Q\left(H A^{-2}\right)^{T}$

$$
\begin{aligned}
& +\mathrm{L}+H A^{-\Delta k} Q\left(H A^{-\Delta k}\right)^{T}+R \\
& =\sum_{j=1}^{\Delta k} H A^{-j} Q\left(H A^{-j}\right)^{T}+R
\end{aligned}
$$

So:

$$
\begin{aligned}
R_{\vartheta_{k} \varphi_{k}} & =H A^{-\Delta k} P(k \mid k-1)\left(H A^{-\Delta k}\right)^{T}-H A^{-\Delta k} Q^{T}-Q\left(H A^{-\Delta k}\right)^{T} \\
& +\sum_{j=1}^{\Delta k} H A^{-j} Q\left(H A^{-j}\right)^{T}+R
\end{aligned}
$$

The covariance of the estimated state is:

$$
\begin{aligned}
P(k \mid k) & =\operatorname{cov}(\tilde{x}(k \mid k))=\operatorname{cov}(\tilde{x}(k \mid k-1)-\bar{K}(k) \tilde{y}(k \mid k-1)) \\
& =P(k \mid k-1)+\bar{K}(k) R_{\tilde{y}_{k} \tilde{y}_{k}} \bar{K}^{T}(k)-R_{x_{k} \tilde{y}_{k}} \bar{K}^{T}(k)-\bar{K}(k) R_{x_{k} \tilde{y}_{k}}^{T} \\
& =P(k \mid k-1)-\bar{K}(k) R_{x_{k} \tilde{y}_{k}}^{T}
\end{aligned}
$$




\section{Simulations and Analysis}

This section compares the performance of the proposed algorithm against the standard KF algorithm without any measurement delays. The target moves with a constant angular velocity $\omega$, the state vector $X=[X, \dot{x}, y, \dot{y}]^{T}$, we can get the system function is:

$$
\dot{X}(t)=A(\omega) X(t)+B(\omega) w(t)
$$

Where:

$$
A(\omega)=\left[\begin{array}{cccc}
0 & 1 & 0 & 0 \\
0 & 0 & 0 & -\omega \\
0 & 0 & 0 & 1 \\
0 & \omega & 0 & 0
\end{array}\right], B(\omega)=\left[\begin{array}{ll}
0 & 0 \\
1 & 0 \\
0 & 0 \\
0 & 1
\end{array}\right]
$$

and $w(t)$ is represented the process noise sequence.

We can get its discrete form as:

$$
X_{k+1}=\left[\begin{array}{cccc}
1 & \sin \frac{\omega T}{\omega} & 0 & -\frac{1-\cos \omega T}{\omega} \\
0 & \cos \omega T & 0 & -\sin \omega T \\
0 & \frac{1-\cos \omega T}{\omega} & 1 & \frac{\sin \omega T}{\omega} \\
0 & \sin \omega T & 0 & \cos \omega T
\end{array}\right] X_{k}+\left[\begin{array}{cc}
T^{2} / 2 & 0 \\
T & 0 \\
0 & T^{2} / 2 \\
0 & T
\end{array}\right] w_{k}
$$

and the covariance of the noise is:

$$
Q=S_{k}\left[\begin{array}{cccc}
\frac{2(\omega T-\sin \omega T)}{\omega^{3}} & \frac{1-\cos \omega T}{\omega^{2}} & 0 & \frac{\omega T-\sin \omega T}{\omega^{2}} \\
\frac{1-\cos \omega T}{\omega^{2}} & T & -\frac{\omega T-\sin \omega T}{\omega^{2}} & 0 \\
0 & -\frac{\omega T-\sin \omega T}{\omega^{2}} & \frac{2(\omega T-\sin \omega T)}{\omega^{3}} & \frac{1-\cos \omega T}{\omega^{2}} \\
\frac{\omega T-\sin \omega T}{\omega^{2}} & 0 & \frac{1-\cos \omega T}{\omega^{2}} & T
\end{array}\right]
$$

If the sensors measure the position of the target only, thus the measurement matrixes of the sensors are:

$H_{i}=\left[\begin{array}{llll}1 & 0 & 0 & 0 \\ 0 & 0 & 1 & 0\end{array}\right]$

The time delay is modeled by a Gaussian distribution, its probabilistic density function (PDF) is:

$p(t)=\frac{1}{\sqrt{2 \sigma^{2}}} \exp \left(-\frac{(t-u)^{2}}{2 \sigma^{2}}\right)$

where $u$ and $\sigma^{2}$ are the mean and variance.

The parameters used in the simulations are described as in table 1 :

Table 2 the Value of the Parameters

\begin{tabular}{ll}
\hline \multicolumn{1}{c}{ Parameter } & \multicolumn{1}{c}{ Value } \\
\hline Initial value state $X_{0}$ & {$\left[\begin{array}{llll}0 & 10 & 0 & 10\end{array}\right]^{T}$} \\
Initial state covariance $P_{0}$ & $\operatorname{diag}([100,100,100,100])$ \\
Simulation steps & 150 \\
Start tracking step & 50 \\
Time duration $T$ & $0.01 \mathrm{~s}$ \\
angular velocity $\omega$ & $0.06 \mathrm{rad} / \mathrm{s}$ \\
process noise parameter $S_{k}$ & 10 \\
measurement noise covariance $R$ & $\operatorname{diag}([5,5])$ \\
mean of time delay & $a T,(a=0,1,2, \mathrm{~L})$ \\
standard deviation of time delay & $T$ \\
minimum time delay & 0 \\
\hline
\end{tabular}


Consider $a=0,5,10,15$, after 50 times Monte-Carlo experiment, the root mean square errors of each variable are as in figures 1-4.

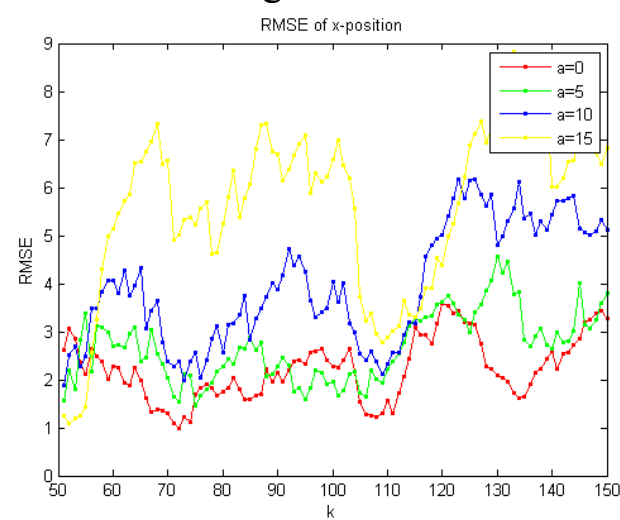

Fig. 1 RMSE of x-position

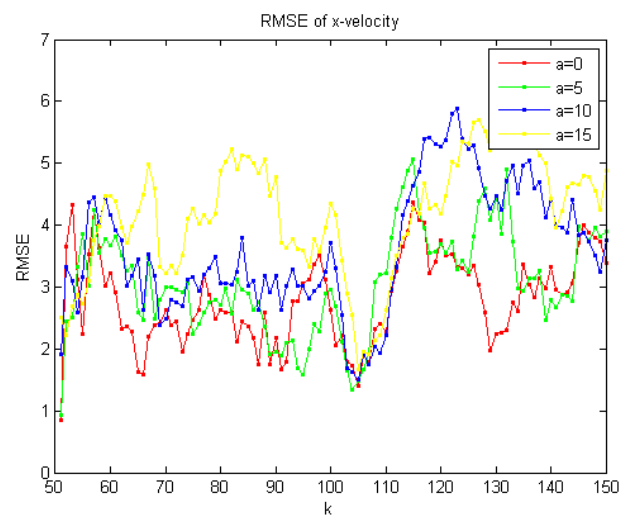

Fig. 3 RMSE of x-velocity

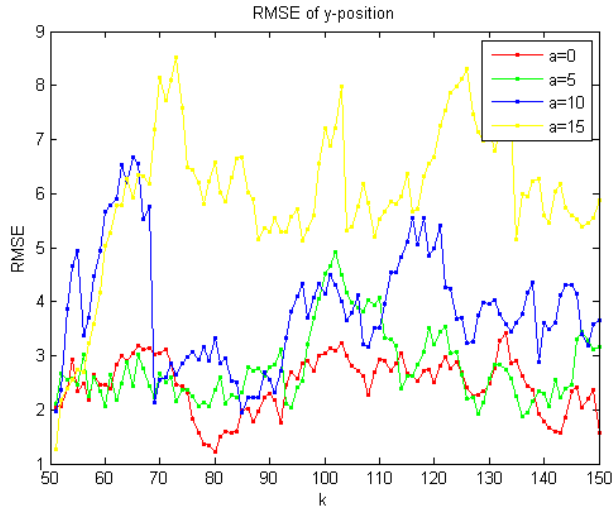

Fig. 2 RMSE of y-position

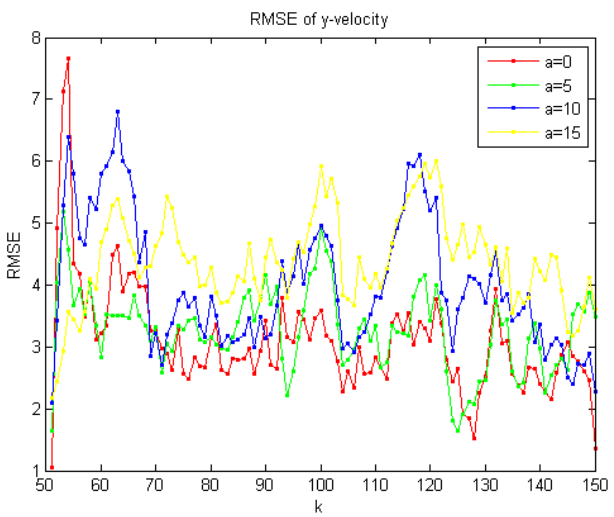

Fig. 4 RMSE of y-velocity

From the figures above, we can see that when the measurement delay is known to the filter, the error of the estimated state is stable. So the proposed algorithm can track the moving target efficiently. Moreover, when $\mathrm{a}=0$, the time delay distribution is a Gaussian distribution with its mean value being zero. So the time delay is the minimum one among the four conditions in statistical significance. And the error is minimal. When $\mathrm{a}=15$, the time delay is the maximum delay, and the corresponding error is maximal. We can get from the figures above that along with the time delay rises, the error rises.

\section{Summary}

In this paper, the measurement delay is considered for the distributed networked Kalman filter. The novel algorithm is proposed to solve the case that the measurement delay is known to the filters. The process of the algorithm has been deduced in detail. Last, Monte-Carlo simulations have been designed, and the results have shown that the proposed algorithm can track the target well. Moreover, along with the time delay rises, the tracking error rises.

\section{References}

[1] Bar-Shalom Y. Update with out-of-sequence measurements in tracking: exact solution. IEEE Trans. Aerosp. Electron Syst. 2012, 38(3): 769-778.

[2] Bar-Shalom Y, Chen. H, Mallick. M. One-step solution for the multistep out-of-sequence measurements problem in tracking. IEEE Trans. Aerosp. Electron Syst, 2004, 40(1): 27-37.

[3] Ubhash Challa, Robin J. Evans, Xuezhi Wang. A Bayesian solution and its approximations to out-of-sequence measurement problems [J]. Information Fusion. 2003, 4: 185-199.

[4] LU Xiao, Wang Wei. Kalman filtering for time-delayed linear systems [J]. Science in China Series F: information Sciences. 2006, 4(49): 461-470. 
[5] Keshu Zhang, X.Rong Li. Optimal Update with Out-of-Sequence Measurements for Distributed Filtering [J]. ISIF 2002.

[6] M.Choi, J.Choi, W.K.Chung. State estimation with delayed measurements incorporating time-delay uncertainty[J]. IET Control Theory and Applications. 2012, 6(15): 2351-2361.

[7] An der Merwe, R. Wan, E.A.Julier, S.I. Sigma-point Kalman filters for nonlinear estimation and sensor-fusion: applications to integrated navigation[C]. Proc. AIAA Guidance, Navigation and Control Conf and Exhibit, Providence, Rhode Island, USA, August 16-19 2004. 